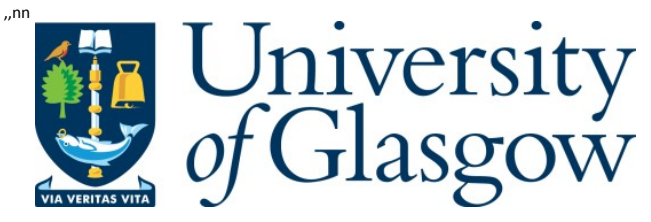

Radwell, N., Clark, T. W., Piccirillo, B ., B arnett, S. M ., and Franke-A rnold, S. (2015) Spatially dependent electromagnetically induced transparency. Physical Review Letters, 114(12), 123603.

Copyright @ 2015 A merican Physical Society

A copy can be downloaded for personal non-commercial research or study, without prior permission or charge

Content must not be changed in any way or reproduced in any format or medium without the formal permission of the copyright holder(s)

http://eprints.gla.ac.uk/105315/

Deposited on: 22 A pril 2015

Enlighten - Research publications by members of the University of Glasgow http://eprints.gla.ac.uk 


\title{
Spatially dependent electromagnetically induced transparency
}

\author{
N. Radwell ${ }^{1 *}$, T. W. Clark ${ }^{1}$, B. Piccirillo ${ }^{2}$, S. M. Barnett ${ }^{1}$ and S. Franke-Arnold $^{1}$ \\ ${ }^{1}$ SUPA, School of Physics and Astronomy, University of Glasgow, Glasgow G12 8QQ, UK \\ 2 Dipartimento di Fisica, Università di Napoli Federico II, \\ Complesso Universitario di Monte S. Angelo, 80126 Napoli, Italy
}

(Dated: March 6, 2015)

\begin{abstract}
Recent years have seen vast progress in the generation and detection of structured light, with potential applications in high capacity optical data storage and continuous variable quantum technologies. Here we measure the transmission of structured light through cold rubidium atoms and observe regions of electromagnetically induced transparency (EIT), using the phase profile as control parameter for the atomic opacity. With q-plates we generate a probe beam with azimuthally varying phase and polarisation structure, and its right and left circular polarisation components provide the probe and control of an EIT transition. We observe an azimuthal modulation of the absorption profile that is dictated by the phase and polarisation structure of the probe laser. Conventional EIT systems do not exhibit phase sensitivity. We show, however, that a weak transverse magnetic field closes the EIT transitions, thereby generating phase dependent dark states which in turn lead to phase dependent transparency, in agreement with our measurements.
\end{abstract}

Introduction: The coherent interaction of light with atoms can cause quantum interference between the excitation amplitudes of different optical transitions, dramatically changing the optical response of a medium. Perhaps the most intriguing example of this is electromagnetically induced transparency (EIT) [1, 2, rendering a medium transparent for resonant probe light when simultaneously exposed to an additional control beam. The anomalous dispersion associated with the 'transparency window' has been exploited for the generation of slow and stopped light [3, 4, and related techniques have led to EIT based quantum memories [5] 9 .

In this letter we report spatially varying optical transparency, achieved by exposing an atomic medium to a single light beam with an azimuthally varying polarisation and phase structure [10]. Such a light mode is structurally inseparable in its polarisation and angular position, an effect sometimes referred to as 'classical entanglement' 1113. If this light is driving a Hanle resonance [14, the left and right-handed circular polarisation components constitute the probe and control for the EIT transition respectively, leading to azimuthally varying atomic dynamics.

It is well established that radially polarised light modes can be focused beyond the diffraction limit [15], and light with an azimuthal polarisation structure has been proposed for enhanced rotational sensing in so-called photonic gears [16. The interaction of atoms with phase structured light has been exploited in a variety of experiments, for EIT systems [17-19] as well as for fourwave mixing [20]. Very recently the first phase-preserving quantum memory has been demonstrated by driving EIT transitions with light entangled in its polarisation and angular position [21, 22] and storing phase-dependent optical information in EIT coherences. In contrast, here we generate spatially varying atomic dark states, rendering the atoms transparent to light at specific angular a)
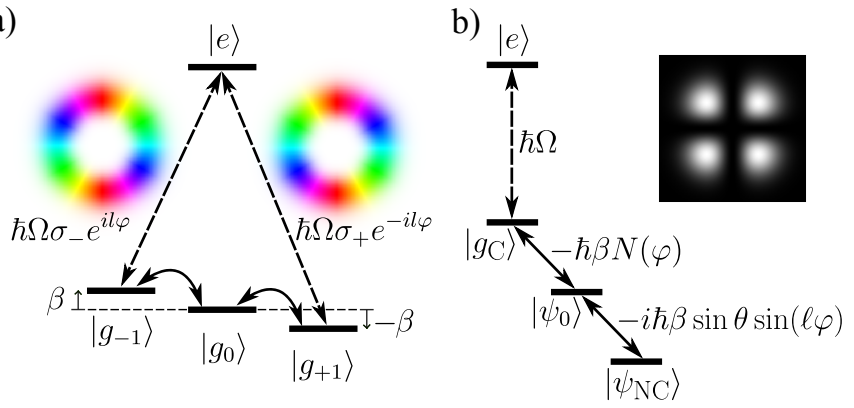

FIG. 1: EIT level scheme with phase dependent driving fields. a) Schematic of the atomic energy levels, optical coupling to the excited state (insets show phase represented as hue colour and intensity as saturation), and weak magnetic coupling between the ground states. b) Alternative level scheme expressed in terms of partially dressed states; the inset is a theoretical simulation of the absorption profile from $\left|\psi_{\mathrm{NC}}\right\rangle$ to $|e\rangle$. Details in main text.

positions. This offers potentially more robust storage, as atoms in dark states are decoupled from the electromagnetic fields. The self-modulation of the incident light beams effectively converts phase into intensity information.

It has been shown theoretically that phase-dependent population dynamics require closed linkages between the excitation amplitudes [23. Here, we demonstrate that a closed loop system can be realised by coupling the EIT ground states with a weak transverse magnetic field, and theoretically describe the phase-dependent interaction, before presenting the realisation.

Theory: We consider the atomic $\Lambda$ system shown in Fig. 1 1a). The atomic ground states, $\left|g_{ \pm 1}\right\rangle$, are Zeeman sublevels of the same hyperfine state with magnetic quantum numbers $m_{\mathrm{F}}= \pm 1$, coupled to the excited state $|e\rangle$ by the left and right $\left(\hat{\sigma}_{ \pm}\right)$circularly polarised components of a probe laser, respectively. In the absence of a mag- 
netic field the process is two-photon resonant, providing ideal conditions for EIT. Any detuning from two-photon resonance results in reduced transparency. Here we are posing the question of whether the transparency of an atomic medium is also affected by a phase difference between the complex excitation amplitudes.

We consider probe light with an electric field amplitude

$$
\hat{\mathbf{E}}(r, \varphi)=\frac{1}{\sqrt{2}} E_{0}(r)\left(\hat{\sigma}_{+} e^{-i \ell \varphi}+\hat{\sigma}_{-} e^{i \ell \varphi}\right),
$$

which is correlated in its spin and orbital angular momentum $(\mathrm{OAM})$. Here $\varphi$ denotes the azimuthal angle and $\ell$ is an integer. A left (right) circularly polarised photon then has a phase dependence $e^{\mp i \ell \varphi}$, corresponding to an OAM of $\mp \ell \hbar, ~ 24,25]$. Each polarisation component has a uniform azimuthal intensity, with a central dark vortex core. As orthogonal polarisations do not interfere, the total beam has the same uniform azimuthal intensity.

This light couples to the atoms via the dipole Hamiltonian

$$
\begin{aligned}
\hat{H}_{D} & =\hat{\mathbf{D}} \cdot \hat{\mathbf{E}}=\frac{\hbar \Omega}{\sqrt{2}}\left(e^{-i \ell \varphi}\left|g_{-1}\right\rangle\left\langle e\left|+e^{i \ell \varphi}\right| g_{+1}\right\rangle\langle e|\right)+\text { h.c. } \\
& =\hbar \Omega\left|g_{C}\right\rangle\langle e|+h . c .
\end{aligned}
$$

where $E_{0}(r)$ is incorporated into the Rabi frequency $\Omega=$ $\Omega(r)$, and in the second line we have introduced the $\varphi$ dependent partially dressed states

$$
\left|g_{\mathrm{C}, \mathrm{NC}}\right\rangle=\frac{1}{\sqrt{2}}\left(e^{-i \ell \varphi}\left|g_{-1}\right\rangle \pm e^{i \ell \varphi}\left|g_{+1}\right\rangle\right)
$$

Note that spontaneous emission causes equal decay into each of the atomic ground states, driving the atom out of the coupling state $\left|g_{\mathrm{C}}\right\rangle$ into the non-coupling state $\left|g_{\mathrm{NC}}\right\rangle$ or the unperturbed atomic state $\left|g_{0}\right\rangle$ after very few absorption-emission cycles. The system exhibits phasedependent coherences, but as $\left|g_{\mathrm{NC}}\right\rangle$ is rotationally symmetric, atomic populations and hence absorption are phase independent. This, of course, was expected, as phase-dependent populations require a closed loop level system [23, 26].

Any magnetic field, as long as it is not aligned with the probe beam propagation direction, will perturb the rotational symmetry. We consider an arbitrary magnetic field $\mathbf{B}=B(\cos \theta \hat{\mathbf{z}}+\sin \theta \hat{\mathbf{x}})$, where the light propagates along $\hat{\mathbf{z}}$ and for simplicity we have chosen $\hat{\mathbf{x}}$ to be the transverse direction of the B-field. The $B_{z}$ component sets the quantisation axis. We are interested in the regime where the associated Zeeman shift is well within the atomic linewidth. The transverse component, instead, couples the lower ground states $\left|g_{ \pm 1}\right\rangle$ and $\left|g_{0}\right\rangle$.

The total interaction Hamiltonian $\hat{H}=\hat{H}_{\mathrm{D}}+\hat{H}_{\mathrm{B}}$ includes, in addition to (2), the magnetic interaction, which in the weak field limit is:

$$
\begin{aligned}
\hat{H}_{\mathrm{B}}= & g_{F} \mu_{B} \hat{\mathbf{F}} \cdot \overrightarrow{\mathbf{B}} \\
= & \hbar \beta\left[\cos \theta\left(\left|g_{+1}\right\rangle\left\langle g_{+1}|-| g_{-1}\right\rangle\left\langle g_{-1}\right|\right)\right] \\
& -\hbar \beta\left[\frac{\sin \theta}{2}\left(\left|g_{0}\right\rangle\left\langle g_{+1}|+| g_{0}\right\rangle\left\langle g_{-1}\right|+\text { h.c. }\right)\right],
\end{aligned}
$$

where $\hat{\mathbf{F}}$ is the total angular momentum operator and we have defined the magnetic parameter $\beta=g_{\mathrm{F}} \mu_{\mathrm{B}} B$. The first term describes the Zeeman shift of $\left|g_{ \pm 1}\right\rangle$ due to $B_{\mathrm{z}}$, whereas the second term describes the mixing of $\left|g_{0}\right\rangle$ with $\left|g_{ \pm 1}\right\rangle$, as illustrated in Fig. 1 1 ). It is instructive to introduce a modified basis set of partially dressed states, combining $\left|g_{\mathrm{NC}}\right\rangle$ and $\left|g_{0}\right\rangle$ :

$$
\begin{aligned}
\left|\psi_{0}\right\rangle & =\frac{1}{N(\varphi)}\left[-\cos \theta\left|g_{\mathrm{NC}}\right\rangle+\sin \theta \cos (\ell \varphi)\left|g_{0}\right\rangle\right] \\
\left|\psi_{\mathrm{NC}}\right\rangle & =\frac{1}{N(\varphi)}\left[\sin \theta \cos (\ell \varphi)\left|g_{\mathrm{NC}}\right\rangle+\cos \theta\left|g_{0}\right\rangle\right]
\end{aligned}
$$

where $N(\varphi)=\sqrt{1-\sin ^{2} \theta \sin ^{2}(\ell \varphi)}$ ensures normalisation. Together with $\left|g_{\mathrm{C}}\right\rangle$ these form a complete basis set, in which the Hamiltonian can be rewritten as

$$
\begin{aligned}
\hat{H}= & \hbar \Omega\left|g_{\mathrm{C}}\right\rangle\left\langle e|-\hbar \beta N(\varphi)| \psi_{0}\right\rangle\left\langle g_{\mathrm{C}}\right| \\
& -i \hbar \beta \sin \theta \sin (\ell \varphi)\left|\psi_{\mathrm{NC}}\right\rangle\left\langle\psi_{0}\right|+\text { h.c. },
\end{aligned}
$$

forming the ladder system shown in Fig. 1p). In this basis $\left|g_{\mathrm{C}}\right\rangle$ couples optically to the excitated state and magnetically to $\left|\psi_{\mathrm{C}}\right\rangle$, which in turn couples magnetically to $\left|\psi_{\mathrm{NC}}\right\rangle$. Importantly, at certain angles $\varphi_{n}=n \pi / \ell$ $(n \in \mathbb{N})$, the state $\left|\psi_{\mathrm{NC}}\right\rangle$ decouples from all fields completely. At those angles, once atoms have decayed into the dark state $\left|\psi_{\mathrm{NC}}\right\rangle$, light can pass unhindered. While EIT is often studied as a function of detuning, here we consider resonant excitation. In this case we find the absorption profile by evaluating Fermi's Golden rule, $T_{i \rightarrow f} \propto \frac{2 \pi}{\hbar}|\langle i|H| f\rangle|^{2}$ :

$$
\begin{aligned}
T_{\psi_{\mathrm{NC}} \rightarrow e} & \propto\left(\frac{2 \pi}{\hbar}\right)^{3}|\hbar \Omega|^{2}|\hbar \beta|^{4}|\sin \theta \sin (\ell \varphi)|^{2} N(\varphi)^{2} \\
& \stackrel{\theta \ll \pi / 2}{\longrightarrow}\left(\frac{2 \pi}{\hbar}\right)^{3}|\hbar \Omega|^{2}|\hbar \beta|^{4}|\theta|^{2}|\sin (\ell \varphi)|^{2}
\end{aligned}
$$

For magnetic fields that have a small transverse component to the probe light propagation, the absorption profile varies sinusoidally with a periodicity of $2 \ell$, shown in the inset in Fig. 1 1 ), whereas for larger $\theta$ the absorption acquires additional structure with twice the periodicity.

Experimental Setup \& Procedure: Our experimental setup utilises q-plates [10], liquid-crystal based retardation waveplates with an inhomogeneous optical axis, which exhibit an azimuthal topological charge $q$. Applying an AC external voltage sets their retardation to $\pi$ 


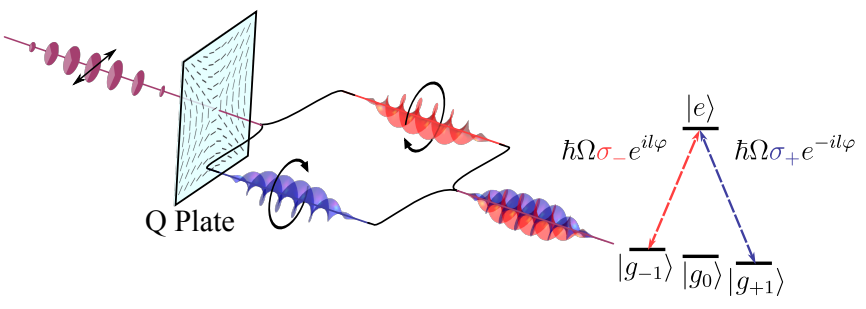

FIG. 2: Probe light generation and differential driving of the atomic transitions. A linearly polarised Gaussian probe beam impinges onto a q-plate, here shown for $q=1$. Its right and left circular polarisation components are encoded with opposite OAM, driving transition amplitudes from the different magnetic sub-levels of the atoms.

27] such that they convert $\sigma_{ \pm}$to $\sigma_{\mp} \exp ( \pm i 2 q \varphi)$. Consequently, linear polarised light generates correlations between polarisation and azimuthal angle of the probe laser, illustrated in Fig. 2 .

The electric field (1) is generated by passing a linearly polarised probe laser through a q-plate with $q=\ell / 2$ along the beam path shown in Fig. 3a). Panels b) and c) show the intensity profile of the probe beam before and after a $q=1$ q-plate. Optional waveplates may be added to alter the polarisation profile. A lens images the far field of the q-plate onto the atoms, which are further imaged onto a CCD camera.

Our experiments are performed on cold, trapped ${ }^{87} \mathrm{Rb}$ atoms, using the hyperfine transition $5^{2} S_{1 / 2}(F=1) \rightarrow$ $5^{2} P_{3 / 2}\left(F^{\prime}=0\right)$. We prepare the sample in a dynamic dark spontaneous force optical trap (SPOT) [28, providing a high density $\left(2 \times 10^{11} \mathrm{~cm}^{-3}\right)$ cloud in the lower $F=1$ ground state.

An experimental run loads a standard MOT for $6 \mathrm{~s}$, reloaded into a SPOT for $250 \mathrm{~ms}$. All trapping lasers are then switched off and the cloud expands for typically $3 \mathrm{~ms}$ to achieve the desired density of $2 \times 10^{11} \mathrm{~cm}^{-3}$, chosen to produce the highest contrast absorption images. The q-plate beam is switched on for $\sim 1 \mathrm{~ms}$ and images are recorded in the presence of atoms $\left(I_{\text {Atoms }}\right)$, in the absence of atoms $\left(I_{\text {Probe }}\right)$ and without lasers $\left(I_{\text {Dark }}\right)$.

In the MOT and SPOT the atoms are exposed to the typical quadrupole magnetic field generated by antiHelmhotz coils. During the atomic expansion we switch off the quadrupole field and add a weak linear magnetic field of $0.1 \mathrm{G}$ by modifying the currents in the compensation coils. This field is predominantly in the longitudinal direction $(\theta \ll \pi / 2)$ with an associated Zeeman shift of less than $1 \%$ of the atomic linewidth.

The transparency profile can be observed directly in the transmitted probe intensity (single shot image shown in Fig 3 d)), but in order to quantify the expected sinusoidal absorption profile we present our data as absorption images. The absorption coefficient is proportional to the optical density, OD $=\log \left(\frac{I_{\text {Probe }}-I_{\text {Dark }}}{I_{\text {Atoms }}-I_{\text {Dark }}}\right)$. At a) Optional
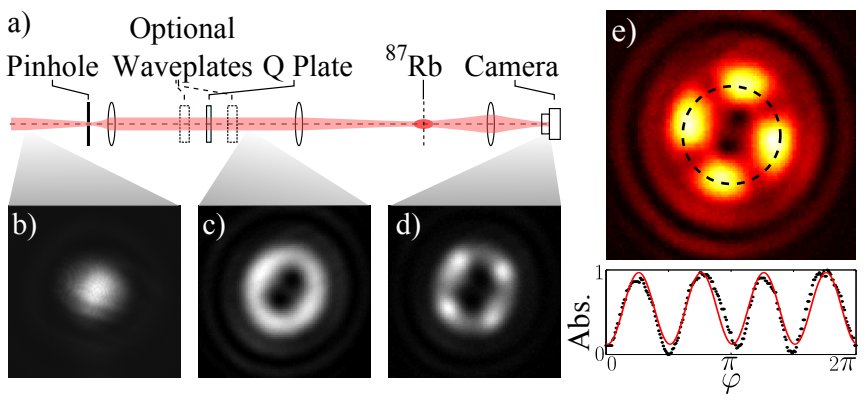

FIG. 3: Experimental setup and initial results. a) Experimental beam path. b) Intensity profile of the input beam, c) of the probe beam generated by the q-plate of $q=1$, and d) after absorption from the atoms. e) Corresponding absorption image and polar plot at radius of maximal contrast.
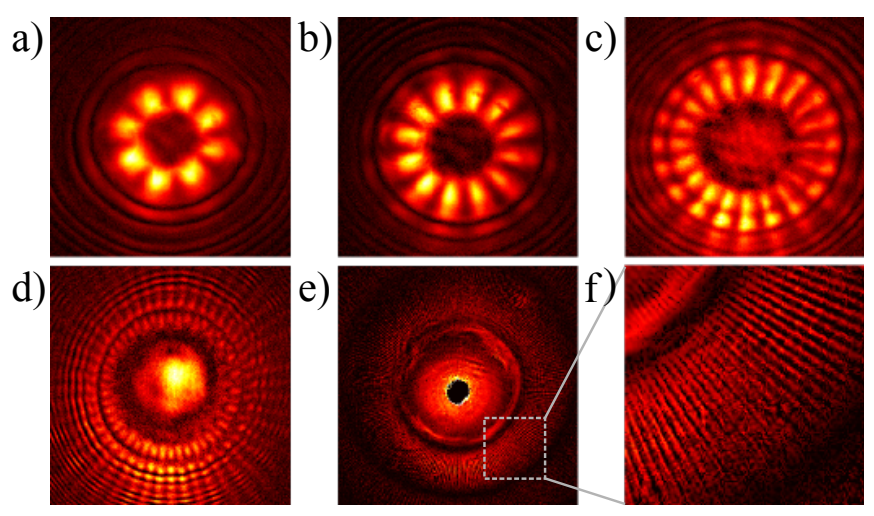

FIG. 4: Absorption patterns for higher q-values, each showing a $4 q$-fold symmetry, for $q=2$ (a), $q=3$ (b), $q=5$ (c), $q=12(\mathrm{~d})$ and $q=100$ (e), with a zoom of the marked section in (f). All absorption patterns arise from single shot images.

low intensity regions the optical density is noisy and/or undefined. We therefore scale our results by a factor of $\sqrt{I_{\text {Probe }}}$ to enhance the meaningful areas of interest (Fig. 3e)). This scaling of course does not affect the azimuthal sinusoidal variation but only the radial intensity profile. Note that bright areas in the absorption image correspond to positions of low light transmission and vice versa.

Results \& Discussion: The procedure outlined above generates a probe beam containing right and left handed circularly polarised components with $\pm 2 \hbar$ units of OAM, which drive transitions from $\left|g_{\mp 1}\right\rangle$, respectively. The absorption profile, shown in Fig. 3e), shows four-fold symmetry, satisfying the $2 \ell$ sinusoidal absorption profile predicted in (7).

Fig. 4 demonstrates that this result holds also for higher rotational symmetries, using different q-plates. All absorption profiles, generated for different q-plates, clearly display the expected $2 \ell$ lobes.

So far we have interpreted spatial transparency as a result of interference between transitions driven by 
a)

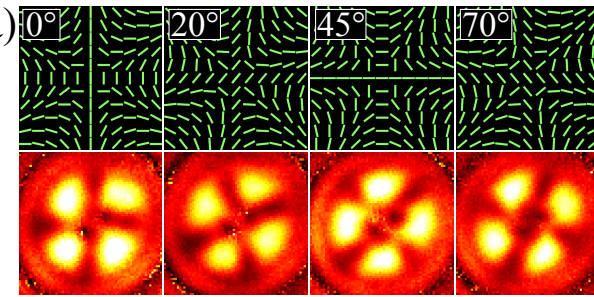

b)

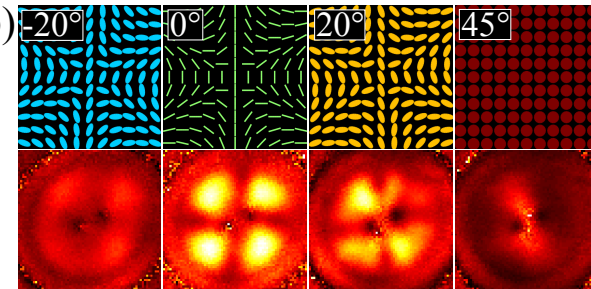

c)

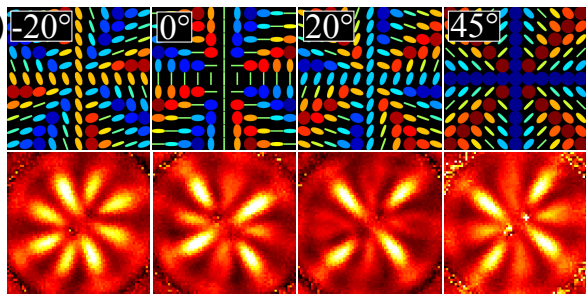

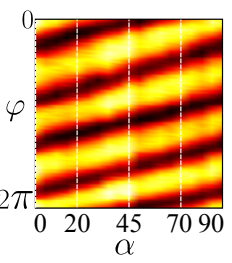
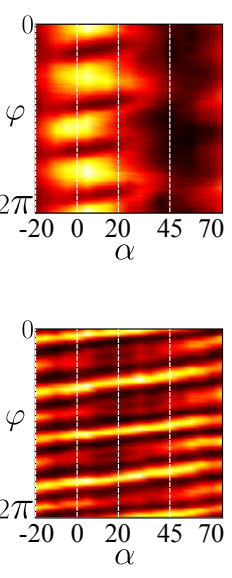

FIG. 5: Absorption profiles as function of input polarisation for a probe beam with $q=1$. a) Rotation of linear input polarisation via a $\lambda / 2$ plate before the q-plate at angle $\alpha$ Probe polarisation profile (top row), and associated measured absorption profile (lower row). To visualise the rotation of the absorption profile with input polarisation we display at the right the polar plot of the absorption profile as a function of $\alpha$, taken every 5 degrees. b) Variation of input ellipticity via a $\lambda / 4$ plate infront of the $\mathrm{q}$-plate. The resulting polarisation profiles vary between a vector vortex beam at $\alpha=0$, associated with maximum contrast in the absorption profile, and a purely circularly polarised beam at $\alpha=45^{\circ}$, generating minimal contrast. c) Adding a $\lambda / 4$ plate after the q-plate changes the symmetry of the observed pattern. No averaging or smoothing procedures have been applied.

the oppositely-phased right and left circular polarisation components. Alternatively, we may consider the overall probe polarisation profile. Our quantisation direction is along the beam propagation. All linear polarisations can then be decomposed into two circular components of equal amplitude, with their relative phase governing the polarisation angle. The probe beams considered for Figs. 3 and 4 have an azimuthally varying phase difference, corresponding to vector vortex beams. The excitation amplitudes interfere only at positions where the local polarisation is aligned with the quantisation axis set by the magnetic field, causing transparency.

We further probe the EIT mechanism by changing the incident polarisation. Based on the system symmetry, one would expect that rotating the linear polarisation before the q-plate simply turns the absorption profile. We test this experimentally by rotating a $\lambda / 2$-plate placed before the q-plate. The observed absorption profiles are

shown in Fig. 5a) for specific $\lambda / 2$ - plate angles. The unwrapped azimuthal profiles displayed on the right panel of Fig. 5 a) combine azimuthal data from 19 individual $\lambda / 2$-plate positions, confirming that the absorption profile follows the input polarisation.

Placing instead a $\lambda / 4$-plate before the q-plate, we can adjust the probe beam's ellipticity from linear to circular. For linear polarisation we retrieve the patterned absorption as before. Purely circular light however, is converted by the q-plate into oppositely circular light and acquires OAM. It will excite only atoms in one of the ground states $\left|g_{ \pm 1}\right\rangle$ and optically pump them into the others, which unlike the state $\left|\psi_{\mathrm{NC}}\right\rangle$ are spatially uniform. The resulting absorption images, shown in Fig. 50), reveal a clear fourlobed pattern at 0 degrees and uniform absorption at about 45 degrees. The unwrapped data, on the right of Fig. $5 \mathrm{~b}$ ), shows the emergence of the absorption structure as a function of the $\lambda / 4$-plate angle.

Fig. 5k), finally, shows the increase of the rotational frequency by placing a $\lambda / 4$-plate after the q-plate, resulting in $4 \ell$ absorption lobes. We note that the atomic system essentially behaves as an 'inverse' linear polariser, with its axis coincident with the quantization axis set by the magnetic field. The petal-like absorption pattern is reminiscent of the petal mode transmission patterns observed by inserting the q-plate between crossed linear polarizers - our 'atomic polariser' however maintains a memory of the incident light.

Conclusion: We have demonstrated spatially structured transparency by probing atoms with vector vortex light. The atomic absorption profile reflects the spatial polarisation variation across the probe beam. We have shown that mixing of the magnetic ground states by a weak magnetic field enables the interference between two excitation amplitudes driven by the different polarisation components of a single probe laser with differential spatial phase profiles. The system contains a spatially varying dark state, whose population in turn resulted in a self-modulation of the incident light beams. Spatial absorption patterns were recorded for a variety of q-plates and input polarisations, showing that the symmetry of the absorption profile is linked to the symmetry of the input polarisation pattern, whereas the contrast of the absorption profile depends on the balance between the excitation amplitudes.

While we have demonstrated spatially dependent EIT for the special case of vortex light beams, the mechanism applies to any light where the circular polarisation components have a different spatial phase profile. Furthermore, while we have used classical light fields, in principle even the circular polarisation components of a single photon could be encoded with different phase profiles, and written into atomic dark states. We hence expect that spatially dependent EIT has applications for the storage of high-dimensional optical information in 
phase-dependent quantum memories. Finally we note that spatially dependent EIT offers the exciting possibility to convert the local correlation between polarisation and azimuthal angle of the probe laser, via the Hanle resonances, into a non-local correlation between the spatial intensity profile of the transmitted light and the atomic population profile.

Acknowledgements: We acknowledge the financial support given by the Leverhulme Trust via the project RPG-2013-386, EPSRC programme grant COAM EP/I012451/1 and for the early work by the European Commission via the FET Open grant agreement Phorbitech FP7-ICT-255914. We thank Lorenzo Marrucci, Fabio Sciarrino, Vincenzo D'Ambrosio and Sergei Slussarenko for useful discussions and above all for the generous provision of various q-plates.

[1] S. E. Harris, J. E. Field, and A. Imamoglu, Phys. Rev. Lett. 64, 1107 (1990)

[2] K.-J. Boller, A. Imamoglu, and S. E. Harris, Phys. Rev. Lett. 66, 2593 (1991)

3 3 L. V. Hau, S. E. Harris, Z. Dutton, and C. H. Behroozi, Nature 397, 594 (1999).

[4] A. V. Turukhin, V. S. Sudarshanam, M. S. Shahriar, J. A. Musser, B. S. Ham, and P. R. Hemmer, Phys. Rev. Lett. 88, $023602(2001)$

[5] D. F. Phillips, A. Fleischhauer, A. Mair, R. L. Walsworth, and M. D. Lukin, Phys. Rev. Lett. 86, 783 (2001)

[6] B. Julsgaard, J. Sherson, J. I. Cirac, J. Fiurásek, and E. S. Polzik, Nature 432, 482 (2004)

[7] A. I. Lvovsky, B. C. Sanders, and W. Tittel, Nat. Photonics 3, 706 (2009)

[8] H. P. Specht, C. Nölleke, A. Reiserer, M. Uphoff, E. Figueroa, S. Ritter, and G. Rempe, Nature 473, 190 (2011)

[9] G. Heinze, C. Hubrich, and T. Halfmann, Phys. Rev.
Lett. 111, 033601 (2013)

[10] L. Marrucci, C. Manzo, and D. Paparo, Phys. Rev. Lett. 96, 163905 (2006)

[11] R. Spreeuw, Found. Phys. 28, 361 (1998)

[12] F. Töppel, A. Aiello, C. Marquardt, E. Giacobino, and G. Leuchs, N. J. Phys. 16, 073019 (2014).

[13] C. Marquardt, E. Giacobino, and G. Leuchs, (2014), arXiv:arXiv:1409.0213v1.

[14] F. Renzoni, W. Maichen, L. Windholz, and E. Arimondo, Phys. Rev. A 55, 3710 (1997)

[15] R. Dorn, S. Quabis, and G. Leuchs, Phys. Rev. Lett. 91, $233901(2003)$

[16] V. D'Ambrosio, N. Spagnolo, L. Del Re, S. Slussarenko, Y. Li, L. C. Kwek, L. Marrucci, S. P. Walborn, L. Aolita, and F. Sciarrino, Nat. Commun. 4, 3432 (2013)

[17] R. Pugatch, M. Shuker, O. Firstenberg, A. Ron, and N. Davidson, Phys. Rev. Lett. 98, 203601 (2007)

[18] D. Moretti, D. Felinto, and J. W. R. Tabosa, Phys. Rev. A 79, 023825 (2009)

[19] L. Veissier, a. Nicolas, L. Giner, D. Maxein, a. S. Sheremet, E. Giacobino, and J. Laurat, Opt. Lett. 38, $712(2013)$

[20] G. Walker, A. S. Arnold, and S. Franke-Arnold, Phys. Rev. Lett. 108, 243601 (2012)

[21] D.-S. Ding, Z.-Y. Zhou, B.-S. Shi, and G.-C. Guo, Nat. Commun. 4, 2527 (2013)

[22] A. Nicolas, L. Veissier, L. Giner, E. Giacobino, D. Maxein, and J. Laurat, Nat. Photonics 8, 234 (2014)

[23] S. J. Buckle, S. M. Barnett, P. L. Knight, M. A. Lauder, and D.T. Pegg, Opt. Acta 33, 1129 (1986)

[24] S. Franke-Arnold, L. Allen, and M. J. Padgett, Laser \& Photonics Reviews 2, 299 (2008).

[25] A. M. Yao and M. J. Padgett, Advances in Optics and Photonics 3, 161 (2011).

[26] D. V. Kosachiov, B. G. Matisov, and Y. V. Rozhdestvensky, J. Phys. B At. Mol. Opt. Phys. 25, 2473 (1992).

[27] B. Piccirillo, V. DAmbrosio, S. Slussarenko, L. Marrucci, and E. Santamato, Applied Physics Letters 97, 241104 (2010).

[28] N. Radwell, G. Walker, and S. Franke-Arnold, Phys. Rev. A 88, 043409 (2013) 